\title{
Identification, expression, and functional analysis of CLE genes in radish (Raphanus sativus L.) storage root
}

\author{
Maria S. Gancheva, Irina E. Dodueva*, Maria A. Lebedeva, Varvara E. Tvorogova, Alexandr A. Tkachenko
} and Ludmila A. Lutova

From The 3rd International Conference on Plant Genetics, Genomics, Bioinformatics and Biotechnology (PlantGen2015)

Novosibirsk, Russia. 17-21 June 2015

\begin{abstract}
Background: Radish (Raphanus sativus L.) is a widespread agricultural plant forming storage root due to extensive secondary growth which involves cambium proliferation and differentiation of secondary conductive tissues. Closely related to the model object Arabidopsis thaliana, radish is a suitable model for studying processes of secondary growth and storage root development. CLE peptides are a group of peptide phytohormones which play important role in the regulation of primary meristems such as SAM, RAM, and procambium, as well as secondary meristems. However, the role of CLE peptides in lateral growth of root during storage root formation has not been studied to date.

Results: In present work we studied the role of CLE peptides in the development of storage root in radish. We have identified 18 CLE genes of radish (RSCLEs) and measured their expression in various plant organs and also at different stages of root development in $R$. sativus and Raphanus raphanistrum -its close relative which does not form storage root. We observed significant decline of expression levels for genes RsCLE1, 2, 11, 13, and 16, and also multifold increase of expression levels for genes RsCLE19, and 41 during secondary root growth in $R$. sativus but not in R. raphanistrum. Expression of RSCLE 2, 19, and 41 in R. sativus root was confined to certain types of tissues while RSCLE1, 11, 13, and 16 expressed throughout the root. Experiments on overexpression of RSCLE2, 19 and 41 or treatment of radish plants with synthetic CLE peptides revealed that CLE19 and CLE2 increase the number of xylem elements, and CLE41 induces the formation of extra cambium foci in secondary xylem. Expression levels of RSCLE2 and 19 strongly decrease in response to exogenous cytokinin, while auxin causes dramatic increase of RsCLE19 expression level and decrease of RsCLE41 expression.

Conclusions: Our data allow us to hypothesize about the role of RSCLE2, 19 and 41 genes in the development of storage root of Raphanus sativus, e.g. RSCLE19 may play a role in auxin-dependent processes of xylem differentiation and RSCLE41 stimulates cambium activity.
\end{abstract}

Keywords: Raphanus sativus, Raphanus raphanistrum, CLE peptides, storage root, Cambium, Xylem

\footnotetext{
* Correspondence: Wildtype@yandex.ru

Department of Genetics and Biotechnology, Saint-Petersburg State

University, Saint-Petersburg 199034, Russia
}

(C) 2016 Gancheva et al. Open Access This article is distributed under the terms of the Creative Commons Attribution 4.0 International License (http://creativecommons.org/licenses/by/4.0/), which permits unrestricted use, distribution, and reproduction in any medium, provided you give appropriate credit to the original author(s) and the source, provide a link to the Creative Commons license, and indicate if changes were made. The Creative Commons Public Domain Dedication waiver (http://creativecommons.org/publicdomain/zero/1.0/) applies to the data made available in this article, unless otherwise stated. 


\section{Background}

The family of CLE (CLAVATA3/ENDOSPERM SURROUNDING REGION) peptide phytohormones includes small (less than $15 \mathrm{kD}$ ) mobile peptides. Being translated as precursors of about 100 amino acid (aa) residues, mature CLE peptides include the only conserved C-terminal CLE domain of 12-14 aa [1]. CLE peptides bind to CLV1-like receptor protein kinases and trigger the poorly studied pathway of signal transduction, which eventually changes the expression level of WOX genes, central regulators of stem cell pools in different meristems [2]. CLE peptides were found in various plant species [3] and also outside of the plant kingdom, in some parasitic nematodes [4]. Arabidopsis thaliana genome contains 32 $C L E$ genes with different spatial and temporal expression patterns $[5,6]$. It is accepted that functions of most CLE peptides in the meristems consist in the negative regulation of stem cell proliferation and thereby maintaining meristem size. An exception from this is a small group of TDIF (TRACHEARY ELEMENT DIFFERENTIATION INHIBITORY FACTOR)-like CLE peptides, which do not suppress cell proliferation in the meristems or even stimulate it in the procambium and cambium [7]. According to [8], CLE peptides which do not suppress meristematic cell proliferation, are combined in the group $\mathrm{B}$, whereas other CLE peptides form group A. B-type CLEs differ from other CLE peptides by the specific amino acid composition of the CLE domain and some features in post-translational modification [7]. Among 32 CLE peptides of Arabidopsis, closely related CLE41, CLE44, and CLE42 belong to the group B [7, 8]. Some Arabidopsis CLE peptides function as central regulators of apical and lateral primary meristems: in particular, CLV3 peptide is required for correct SAM development [9]; CLE40 has similar function in RAM [10]; CLE41/CLE44 controls the development of procambium and cambium [11]. In addition, several CLE peptides regulate other types of meristems, e.g. A-type CLEs MtCLE13 and PsCLE13 regulate development of nodule meristems in Medicago truncatula and Pisum sativum respectively $[12,13]$. Moreover, CLE peptides are supposed to regulate other developmental processes, like early embryogenesis [14] or vessel development [11, 15]. Taking all of this into account, we can hypothesize that CLEs may function as regulators of storage root development in radish.

Radish (Raphanus sativus L., $2 n=2 x=18$ ) is an important vegetable crop because of its edible storage root. Several variations of cultivated radish are known, e.g. cherry radish (R. sativus L. var. radicola Pers), oil radish (R. sativus L. var. oleifera), feed radish ( $R$. sativus L. var. caudatus), black radish ( $R$. sativus $\mathrm{L}$. var. niger), and large root radish, or daikon ( $R$. sativus $\mathrm{L}$. var. longipinnatus Bailey). According to most opinions, $R$. sativus $\mathrm{L}$. was originated from wild radish $R$. raphanistrum $\mathrm{L}$. or was derived by hybridization between $R$. maritimus and $R$. landra $[16,17]$. In recent years, the RadishBase [18], a genomic database of radish, was developed [19], and comprehensive analysis of expressed sequence tags from cultivated and wild radish was performed [20]. A genetic collection of R. sativus L. var. radicola Pers is maintained in Saint-Petersburg State University (Russia) since 1970. This collection was derived from single plants belonging to three radish cultivars (Saxa, Virovskyi Belyi, Ledianaya Sosulka) by inbreeding during more than 40 generations. At present, the collection includes 33 self-compatible highly inbred lines; some of them demonstrate different morphological abnormalities (e.g. dwarfism, agravitropic growth, tumor formation etc.) [21, 22].

Unlike its close relative Arabidopsis thaliana, R. sativus has a peculiar developmental feature, namely the formation of a storage root (so called crop-root). Therefore, radish is a perspective model to study mechanisms of storage root development. It is known that radish crop-root originates from the hypocotyl and upper part of root [23] due to the secondary thickening as a result of cambium activity [24, 25]. The secondary thickening involves proliferation of vascular cambium and differentiation of secondary xylem and phloem inwards and outwards from cambium. In case of radish the proportion of phloem and xylem differentiation is shifted towards xylem (so called xylem-type storage root). As a result, mature radish storage root is composed of a large zone of secondary xylem tissue surrounded by a narrow cambial zone, band of secondary phloem, and outer layer of secondary cortex derived from pericycle [25]. Zone of secondary xylem in radish storage root is quite clearly separated into central and periphery parts. Periphery part of secondary xylem zone includes vessels surrounded by rows of small-cell thick-walled parenchyma cells which perform predominantly mechanical function (mechanical parenchyma); vessels and mechanical parenchyma form bands, separated by wide radial rays of thinwalled parenchyma cells filled with starch grains [26]. Central part of secondary xylem in the root of $R$. sativus includes more rare vessels lying in the mass of the thinwalled storage parenchyma (Fig. 1b). Some researchers $[26,27]$ noted that zone of secondary xylem in radish storage root (mainly an inner region of secondary xylem) also includes numerous little foci of cambium-like secondary meristem (so called "meristematic foci") which are maintained for a limited time and give rise to a small number of tertiary conductive elements. On the other hand, $R$. raphanistrum, presumable ancestor of $R$. sativus [16, 17] demonstrate less extensive secondary thickening and does not form storage root. The main differences in the anatomical structure of $R$. sativus and $R$. raphanistrum are less extensive zone of secondary xylem and significantly fewer cells of the storage parenchyma in the root of $R$. raphanistrum (Fig. 1). 


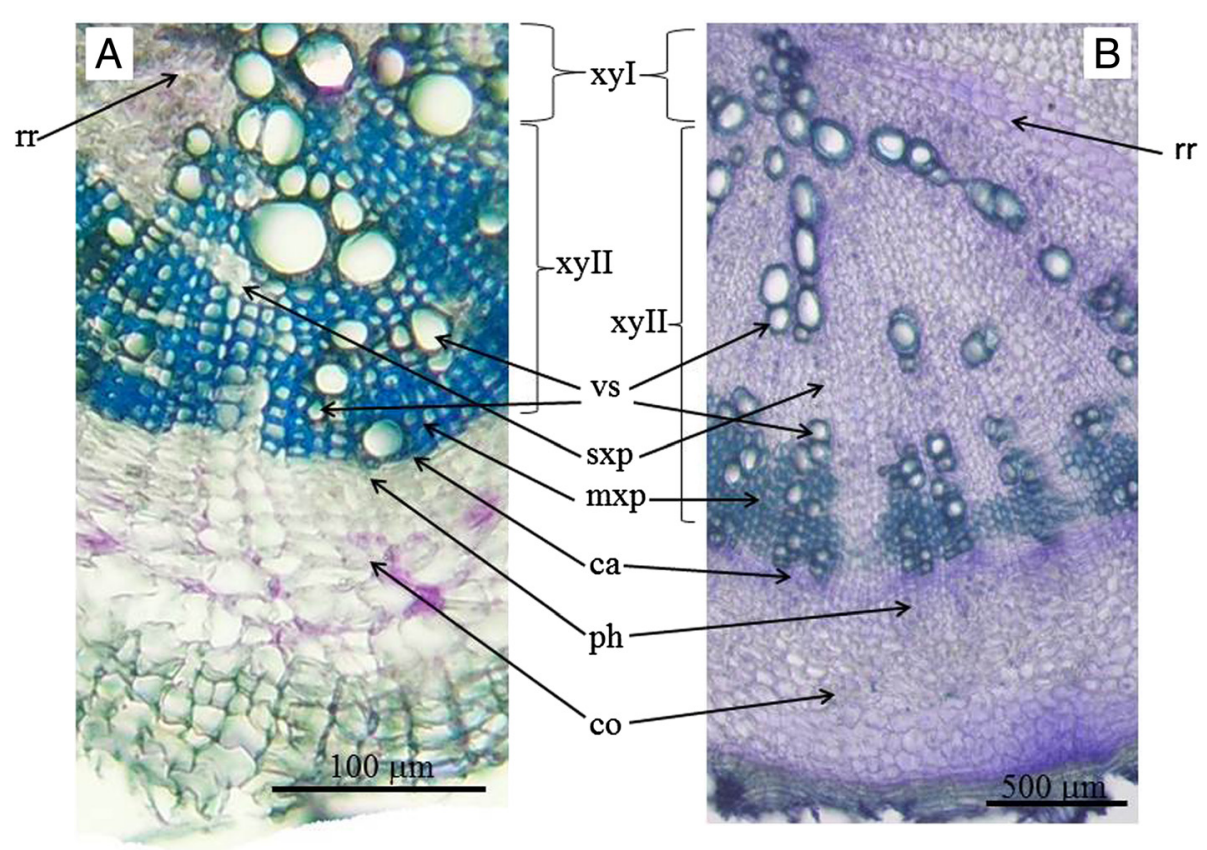

Fig. 1 Comparison of the anatomical structure of Raphanus raphanistrum (a) and Raphanus sativus (b) roots (30 day old plants). xyl—primary xylem, xyll—secondary xylem, ca—cambium, ph—phloem, co—cortex, vs—vessels, mxp—-mechanical xylem parenchyma, sxp—storage xylem parenchyma, rr-radial ray

The main goal of our investigation was determining of the role of CLE peptides in the development of storage root in radish. In the present work we identified Raphanus sativus RSCLE genes based on their homology with corresponding Arabidopsis thaliana AtCLE genes. Then, we analyzed the expression of RsCLE genes in different parts of seedling, and also in the roots at different stages of development in $R$. sativus which forms storage root and in $R$. raphanistrum which does not. In addition, we studied the effect of overexpression of certain RsCLE genes of A- and B-types as well as the effect of exogenously applied CLE peptides on radish storage root development. Our data suggest the involvement of RsCLE2, 19, and 41 genes in the development of radish storage root. Finally, we studied the effect of exogenous cytokinin and auxin on the expression of several A- and B-type RsCLE genes. The results obtained indicate that CLE-peptides, cytokinins and auxins may interact during the development of radish storage root.

\section{Results and discussion}

\section{Identification of Raphanus sativus CLE (RsCLE) genes}

The genome of Arabidopsis thaliana contains 32 CLE genes [1], and each of them has its own unique expression pattern $[5,6]$. We identified radish homologues of the most part of Arabidopsis AtCLE genes whose expression, according to [6], was observed in the root (except for AtCLE6, 7, and 44). In total we have identified 16
RsCLE genes homologous to Arabidopsis A-type CLE genes (RsCLE1, 2, 4, 5, 11, 12, 13, 16, 17, 19, 20, 22, 25, 26, 27, and 40) and 2 RsCLEs that are homologous to Arabidopsis B-type CLE genes (RsCLE41, and RsCLE42). CDS (coding sequences) of RsCLEs identified demonstrated 72.9-90.7 \% of identity with corresponding AtCLE genes (Table 1) and also with CLE-like sequences of Brassica rapa [28]. However, we failed to find radish homologues of AtCLE6, AtCLE7, and AtCLE44 genes: all primer sets that were designed based on corresponding genes of Arabidopsis did not give any PCR product on radish DNA or anneal to other closely related RsCLEs. Therefore, we suppose that there are no homologues of CLE6, CLE7, and CLE44 genes in Raphanus sativus genome. The sequences homologous to AtCLE6, AtCLE7, and AtCLE44 genes are also absent among identified Brassica rapa sequences [28].

Predicted sequences of CLE domains of most of RsCLEs are similar to those of AtCLEs. Exceptions are RsCLE11, 17, 19, 22, 26, and 27 whose CLE domains presumably differ from Arabidopsis in one or two amino acids (Table 1). Like AtCLE genes, RsCLEs gene sequences are short, their coding sequence lengths are 240-330 bp. Most of RsCLEs, like AtCLEs, lack introns, with the exception of RsCLE40, which has two introns like its homologue AtCLE40 [10].

According to previous data, substitution of the glycine residue in the sixth position of CLE domain has the 
Table 1 Identified Raphanus sativus CLE (RSCLE) genes

\begin{tabular}{|c|c|c|c|c|}
\hline Gene & Length of CDS, bp & Identity with CDS of Arabidopsis genes, $\%$ & CLE-domain sequence ${ }^{\mathrm{a}}$ & GenBank accession number \\
\hline RsCLE1 & 234 & 80,3 & RLSPGGPDPRHH & KF965525 \\
\hline RSCLE2 & 240 & 84,6 & RLSPGGPDPQHH & KF965526 \\
\hline RsCLE4 & 249 & 87,6 & RLSPGGPDPRHH & KF965533 \\
\hline RSCLE5 & 246 & 80,1 & RVSPGGPDPQHH & KF965527 \\
\hline RsCLE11 & 297 & 84,0 & RㅡVSGP奋PLHH & KF965528 \\
\hline RsCLE12 & 366 & 79,0 & RRVPSGPNPLHH & KT803936 \\
\hline RsCLE13 & 315 & 74,5 & RLVPSGPNPLHH & KF965529 \\
\hline RSCLE16 & 306 & 75,6 & RLVHTGPNPLHN & KF965534 \\
\hline RsCLE17 & 280 & 75,2 & RV므TGPNPLHN & KF965535 \\
\hline RsCLE19 & 228 & 86,4 & RIIPTGPNPLHN & KF965530 \\
\hline RsCLE20 & 255 & 85,1 & RKVKTGSNPLHN & KF965536 \\
\hline RsCLE22 & 306 & 82,4 & RRVFTGPNP$\underline{\mathbf{S}}$ H $\underline{\mathbf{S}}$ & KF965537 \\
\hline RsCLE25 & 246 & 90,7 & RKVPNGPDPIHN & KF965538 \\
\hline RsCLE26 & 346 & 74,6 & RKVPRGSIDPIHN & KT803934 \\
\hline RsCLE27 & 282 & 72,9 & RㅁVSSGPDPLHN & KT803935 \\
\hline RsCLE40 & 261 & 84,7 & RQVPTGSDPLHH & KF965539 \\
\hline RsCLE41 & 300 & 86,7 & HEVPSGPNPISN & KF965531 \\
\hline RsCLE42 & 255 & 84,3 & HGVPSGPNPISN & KF965532 \\
\hline
\end{tabular}

amino acid residues which differ from the amino acids in the CLE domain of Arabidopsis are marked by bold underscored text

most pronounced effect on the function of CLE peptides in Arabidopsis [29]. The glycine at sixth position is highly conserved in CLE-domains of all Arabidopsis CLE peptides; the only exception is AtCLE27 that has cysteine at sixth position of CLE-domain. However, all identified radish CLEs contain conserved glycine residue in the sixth position of CLE-domain.

\section{qRT-PCR analysis of RsCLE genes expression}

Using qRT-PCR method, we analyzed RsCLE genes expression in different organs of $R$. sativus and $R$. raphanistrum seedlings. Most of RsCLEs expressed in all parts of plants, but some of them were expressed in a certain plant organ only, e.g. RsCLE1, RsCLE2, and RsCLE13 demonstrated root-specific expression (Additional file 1: Table S1 and Additional file 2: Figure S1). According to literature data [5, 6] corresponding AtCLE genes have similar expression pattern in seedlings. Among studied RsCLEs, expression of RsCLE16 was the strongest in root and hypocotyl of $R$. sativus seedlings, whereas RsCLE4 and RsCLE17 were the most weakly expressed genes (Additional file 2: Figure S1). Expression patterns of some RsCLEs slightly differed in $R$. sativus and $R$. raphanistrum seedlings-e.g. RsCLE19 expressed at high level in the root of $R$. sativus but not in the root of $R$. raphanistrum.

Then we analyzed the expression of RsCLEs in the root and hypocotyl in two different lines of $R$. sativus and in
$R$. raphanistrum at different developmental stages: 7-day old seedling, 15- and 30-day old plants (stages 1, 2, 3, correspondingly). In $R$. sativus 15 -day old plants at four leaves stage, storage root formation is started through the extensive root thickening, in 30-day old plants at rosette stage root thickening reaches the maximum [25]. In contrast to $R$. sativus, $R$. raphanistrum does not form a storage root, and therefore demonstrates less pronounced root thickening at the same developmental stages which gave us the reason to check whether $R$. sativus and $R$. raphanistrum differ in expression levels of RsCLEs

We revealed that expression levels of some RsCLEs were significantly increased or decreased in the roots and hypocotyls of 15- and 30-day old plants of both analyzed $R$. sativus lines, but not in the roots and hypocotyls of $R$. raphanistrum (Fig. 2a, b, and Additional file 3: Figure S2). Genes, whose expression increases tenfold and more in thickening storage root of $R$. sativus, included one A-type CLE gene (RsCLE19) (Welch's $t$-test $p$-value $<0.001 ; n=3)$, and one B-type CLE gene-(RsCLE41) (Welch's $t$-test $p$-value $<0.001 ; n=$ $3)$. At the same time, expression levels of other five A-type CLE genes-RsCLE1, 2, 11, 13, and 16 decreased significantly (Welch's $t$-test $p$-values 0.0153, $0.0359,0.0045,<0.0001,<0.0001$, respectively; $n=3$ ) during root thickening in $R$. sativus plants (Fig. 2a, b, and Additional file 3: Figure S2). 

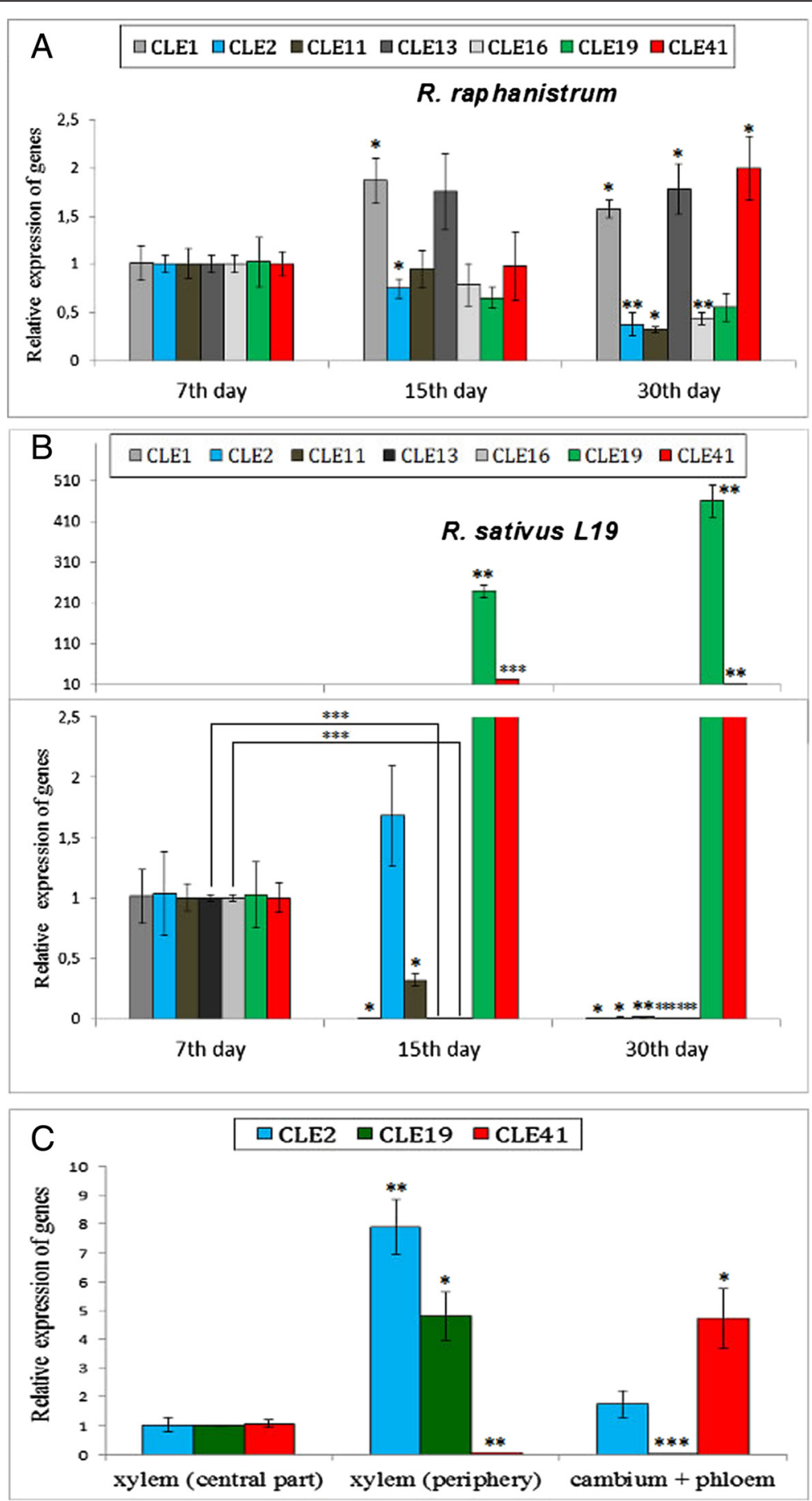

Fig. 2 qRT-PCR analysis of RsCLE genes expression. a, b RsCLE genes expression in Raphanus raphanistrum (a) and Raphanus sativus (b) roots (line 27) at different stage of development. Expression levels are shown relative to the expression found in the root of 7-day old seedlings. $\mathbf{c}$ RsCLE genes expression in different tissues of Raphanus sativus storage root. Error bars indicate standard deviation of three technical repeats. $(P<0.05-*$, $\left.P<0.01{ }^{* *}, P<0.001{ }^{* * *}\right)$

For more detailed expression analysis of RsCLE1,2, $11,13,16,19$, and 41 we dissected several zones in R. sativus storage root (central (1) and periphery (2) parts of secondary xylem, and also phloem + cambium (3)) and then analyzed RsCLEs expression in these zones. We found that RsCLE1, 11, 13, and 16 are expressed in all tissues across radish root, while RsCLE2, 19, and 41 have zone-specific expression pattern. RsCLE2 and 
19 are expressed specifically in secondary xylem, and RsCLE41 is expressed in the phloem and cambium zones. It is interesting that the expression of all studied RsCLEs was revealed in secondary meristem foci which are initiated in central part of xylem in radish storage root (Fig. 2c).

According to our results, three RsCLEs (RsCLE2, 19, and 41) specifically express in certain tissues that provide secondary growth of root (RsCLE2 and 19 were expressed in secondary xylem, RsCLE41 was expressed in phloem and cambium). Moreover, dramatic change of their expression levels was associated with the beginning of extensive root thickening in $R$. sativus. So, we suppose that these genes may participate in the regulation of storage root development.

In Arabidopsis, 23 of 29 AtCLE genes encoding A-type CLE peptides are also expressed in roots, and some of them demonstrate tissue-specific expression pattern [5, 6]. All Arabidopsis genes encoding B-type CLE peptides (e.g. AtCLE41) are expressed in the vascular tissues of all plant organs including the root [30, 31]; AtCLE19 and BnCLE19 genes of Arabidopsis and Brassica roots are expressed specifically in pericycle cells facing the protoxylem poles [32], and AtCLE2-in the primordial of lateral roots [6].

\section{Effect of altered expression levels of RsCLEs and of treatment with exogenous CLE peptides on radish storage root development}

Many investigators have studied the changes in different aspects of plant development caused by altered expression levels of $C L E$ genes, or by treatment of plants with exogenous CLE peptides. Most of such studies are focused mainly on SAM and RAM activity [6, 7, 33-35], some of them also consider cambium activity and vascular system development $[7,8,11]$. These experiments revealed that B-type CLE peptides (CLE41, 42, and 44 in Arabidopsis) stimulate proliferation of cambium cells via activation of WOX4 gene expression, and also inhibit their differentiation into xylem. On the other hand, it is obvious that some A-type CLE peptides also take part in the development of vascular system; e.g. overexpression of CLE19 gene in Arabidopsis and Brassica stimulated the differentiation of xylem, leading to the formation of xylem "islands" in the flower organs [32]; AtCLE10 inhibits vessel formation in Arabidopsis roots via repression of the expression of two type-A Arabidopsis Response Regulators (ARRs), ARR5 and ARR6, whose products act as negative regulators of cytokinin signaling [15].

Thus, A- and B-type CLE peptides may control the balance between cambium cells proliferation and differentiation of xylem. Nevertheless, simultaneous overexpression of A- and B-type CLE genes, or plant treatment by CLE peptides from both groups leads to more pronounced stimulating effect on cambium cell proliferation than overexpression of only B-type CLE genes [8]. Therefore, interaction of A- and B-type CLEs in the control of vascular system development and root secondary growth is more complex than simple antagonism.

We have studied the effect of overexpression of several RsCLEs, encoding A-type (RsCLE2 and 19) and B-type (RSCLE41) CLE peptides on secondary root structure in radish. We also treated $R$. sativus and $R$. raphanistrum plants by synthetic CLE peptides CLE2, CLE19, and CLE41. Both experiments provided very similar results: plants with shifted quantity of a certain CLE peptide in the root (resulted from CLE overexpression or treatment by exogenous CLE) had altered development of storage root tissues (Fig. 3).

First, roots of R. sativus 15- and 30-day old plants with overexpression of RsCLEs or after treatment by CLE peptides demonstrated altered quantity of certain type of secondary xylem elements-mechanical xylem parenchyma, which is normally adjacent to vessels in mature storage root of radish. Unexpectedly, number of secondary xylem vessels remained unchanged (Fig. 2).

In the root of the radish plants with RsCLE41 overexpression or treated by CLE41 the cells of the mechanical xylem parenchyma were absent. The same effect we observed in the radish roots with overexpression of RsCLE2 or treated by CLE2 peptide. Conversely, RsCLE19 overexpression or CLE19 peptide treatment increased the number of mechanical xylem parenchyma cells (Welch's $t$-test $p$-value $=0.0092 ; n=4$ ). Therefore, A-type CLE peptides CLE2 and CLE19 presumably play different roles in the differentiation of secondary xylem elements, mainly of mechanical xylem parenchyma. Earlier, the negative effect of CLE41 on xylem differentiation was observed in Arabidopsis [7, 8]; Fiers et al. also revealed stimulatory effect of CLE19 on formation of extra xylem islands in Arabidopsis [32]; but the effect of CLE2 on xylem differentiation was not previously observed.

Secondly, radish roots with overexpression of RsCLE41 or treatment by CLE41 peptide had increased number of meristematic foci in a central part of secondary xylem. According to literature data $[26,27]$, during the formation of storage root in radish some xylem parenchyma cells proliferate giving rise to meristematic foci-small areas of secondary cambium capable to divide and differentiate into phloem and xylem cells. In the roots with overexpression of RsCLE41 or treated with CLE41 peptide these "meristematic foci" are enlarged and include small thin-walled cells, similar to cells of cambium, young vessels surrounding cells thick-walled xylem parenchyma cells and also phloem cells (Fig. 2). Therefore, in the storage root of radish, CLE41 can stimulate the proliferation of not only regular cambium, but also of cambium cells in the meristematic foci. 


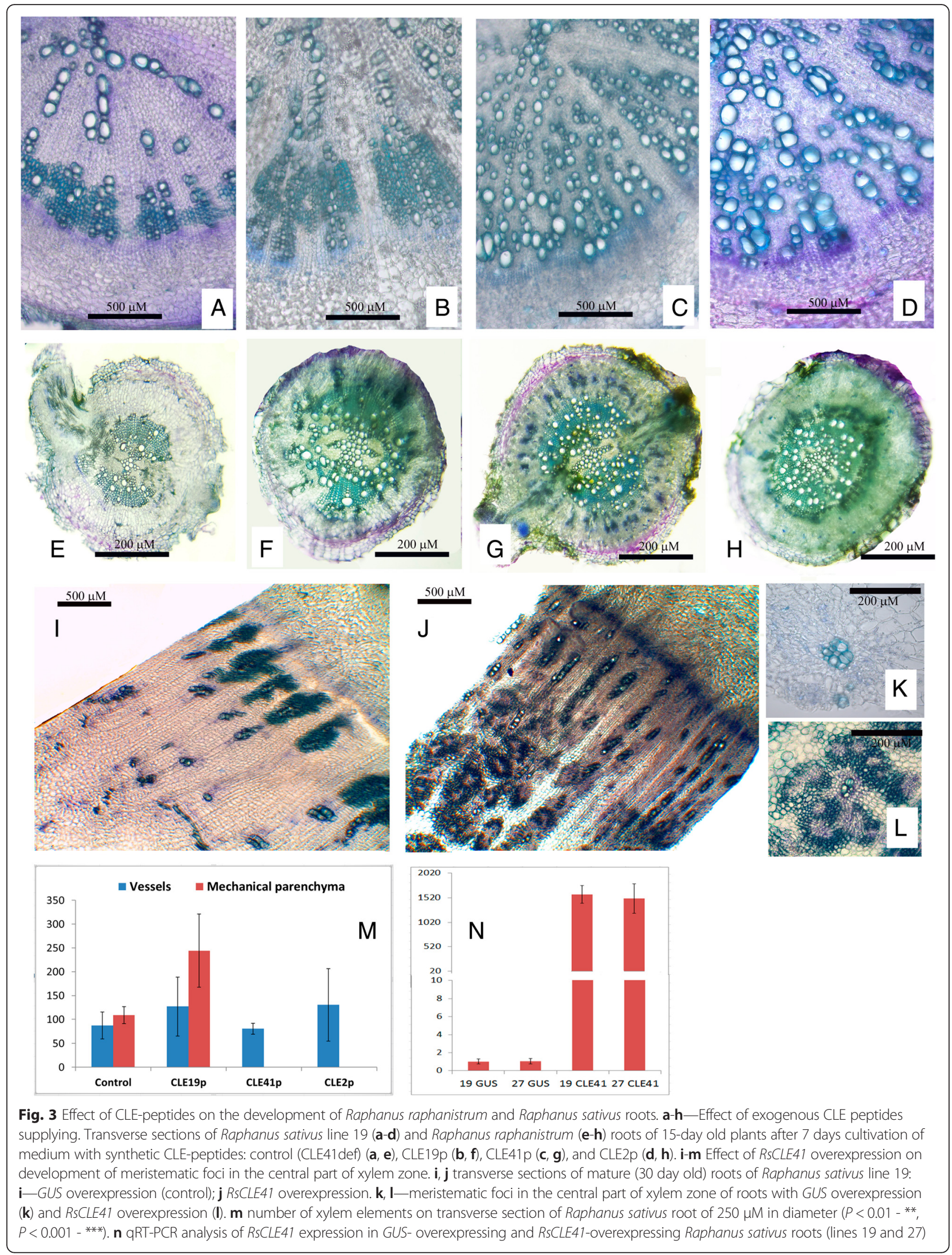


We also treated $R$. raphanistrum roots with exogenous CLE-peptides. In contrast to $R$. sativus, the effect of CLE-peptides on $R$. raphanistrum root structure was less pronounced, however, the general trend of the changes was the same. CLE19p slightly increased the number of mechanical xylem parenchyma, but there was no statistically significant difference in the number of xylem elements between control and CLE19-treated plants. CLE41 increased cambium cells number, however, no extra cambium foci were observed in the secondary xylem of CLE41-treated as well as in control $R$. raphanistrum plants. Treatment with CLE2p did not cause any changes in the number of xylem elements in $R$. raphanistrum roots. We speculate that different effects of CLE peptides on root structure of $R$. sativus that forms storage roots and $R$. raphanistrum may result from the differences in regulation of secondary root development in these radish species. Moreover, these two species might have initially different levels of other plant hormones interacting with CLE-peptides, which also could lead to different responses to exogenous CLE-peptide treatment.

\section{Influence of exogenous cytokinin and auxin on expression of RsCLE genes}

It is well known that auxins and cytokinins are two main groups of phytohormones regulating the development of vascular system and secondary thickening of root. In the primary root of Arabidopsis these hormones demonstrate complementary patterns of distribution: auxins are concentrated in the differentiated xylem, while cytokinins are present in the cambium and phloem. Complementary auxin and cytokinin distribution is believed to be required for the proper development of the vascular system and to result from of cytokinin-dependent control of polar auxin transport and auxin-dependent repression of cytokinin signaling [36]. Cytokinins are necessary for induction of cambium in procambium and also cambium cell proliferation [37], auxin is also needed for proliferation of cambium-probably due to auxindependent control of WOX4 gene expression [38]. Auxin transport and signaling components also play a key role in vascular cell specification [39].

So, there are two groups of regulators that control the development of vascular system and root secondary thickening-auxins and cytokinins along with CLEpeptides. It is probable that they can have some common targets-e.g. WOX4 gene, and thereby, they may interact.

Previously, some data on the interaction between cytokinins and CLE-peptides or auxins and CLE peptides have been reported. In $M$. truncatula, it was discovered that synthetic cytokinin BAP has a stimulating effect on the expression of MtCLE12 and MtCLE13 genes which are central regulators of symbiotic nodule formation [12]. Conversely, Arabidopsis A-type CLE peptide AtCLE10 can stimulate cytokinin signaling by negative regulation of A-type ARR gene expression [15]. In rice, A-type CLE gene OsCLE48 expression was induced by exogenous application of IAA [40]. In Arabidopsis lateral root formation some AtCLEs were up- or down-regulated by different hormones-auxin, ABA, brassinosteroids, salicylic acid and jasmonic acid, as well as by nutrients and stress [35]. However, almost nothing is known about the mechanism of interaction of CLE-peptides and other hormones.

We measured the effect of exogenous auxin (NAA) and cytokinin (BAP) treatment on the expression of RsCLEs in upper part of the root and lower part of hypocotyl of $R$. sativus seedlings. We observed different expression dynamics for analyzed A-type (RsCLE2, 5, 19) and B-type $(R s C L E 41,42)$ RsCLEs in response to cytokinin treatment. Expression of A-type RsCLEs strongly decreased (ten times and more) after BAP treatment, even as early as $0.5 \mathrm{~h}$ after the treatment, whereas the expression of group B RsCLEs in response to BAP was not significantly altered (Fig. 4a). We also observed different expression dynamics for the same A- and B-type $R s C L E s$ in response to treatment by auxin: expression levels of A-type RsCLEs increased (tenfold and more for RsCLE19, less than tenfold for RsCLE2 and RsCLE5), while expression of B-type genes (RsCLE41 and 42) decreased dramatically (Fig. 4b).

Therefore, the same A- and B-type RsCLEs analyzed demonstrated quite different dynamics of expression in response to auxin and cytokinin: cytokinin seems to repress the expression of A-type RsCLEs but it did not influence the B-type RsCLEs; conversely, auxin seems to stimulate expression of A-type gene RsCLE19 and negatively regulate both $\mathrm{B}$-type $R s C L E s$.

Our results coincide with data on the role of cytokinin and B-type CLEs, and also auxin and A-type CLEs in the development of vascular system. It is known that auxin [41] and A-type CLEs such as AtCLE19 [32] stimulate xylem differentiation, whereas cytokinin [37] and B-type CLEs [11, 30] are necessary for cambium development. Taking that into account, we can suppose that these three groups of phytohormones can interact during the formation of plant vascular system and the development of radish storage root.

\section{Conclusions}

Mechanisms underlying the formation of the storage root are poorly understood. In our study, we investigated the role of CLE peptides in the development of storage root of radish. Our data indicate that some CLE peptides such as CLE19, 41 and 2 may play a role in this process. Thus, the existing ideas about the functions of these peptides in plant development can be extended. Our findings on the effect of cytokinin and auxin on expression of genes encoding A- and B-types CLE peptides 


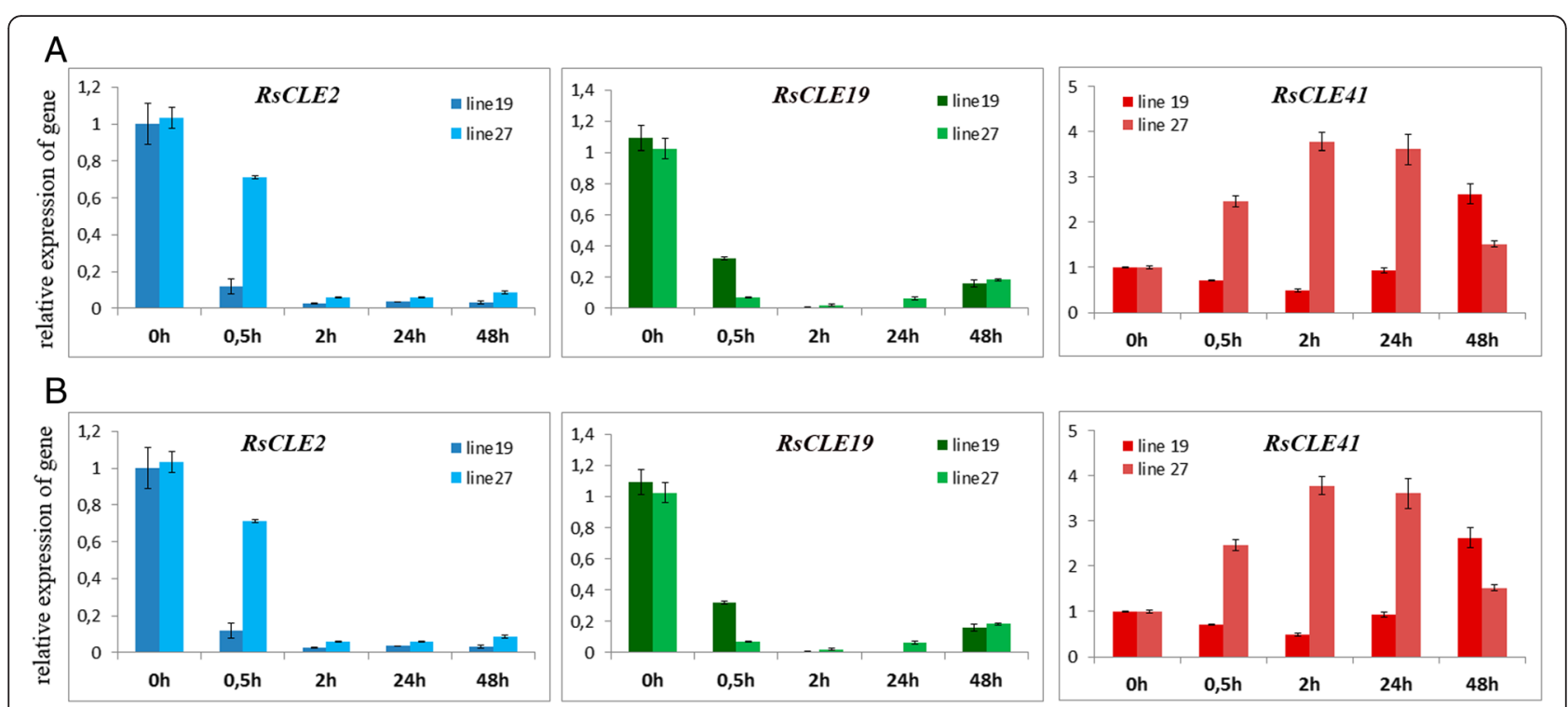

Fig. 4 Effect of exogenous cytokinin (BAP, $10 \mathrm{mM})(\mathbf{a})$ and auxin (NAA, $10 \mathrm{mM})(\mathbf{b})$ on expression of RsCLE2, 19 and 41 genes in upper part of root of Raphanus sativus seedlings. Error bars indicate standard deviation of three technical repeats. $\left(P<0.05-*^{*}, P<0.01-{ }^{* *}, P<0.001-*^{* *}\right)$

allow to suppose the interaction between different groups of phytohormones in the development of storage root of radish.

\section{Methods}

\section{Plant material}

Raphanus raphanistrum and two related inbred lines from a genetic collection of Raphanus sativus [21] 19 and 27 , were used in this study.

\section{Plant growing conditions}

For qRT-PCR and microscopy studies $R$. sativus and $R$. raphanistrum plants were grown in the ground. For transformation by Agrobacterium rhizogenes 7-day old $R$. sativus seedlings grown under aseptic culture conditions were used. For treatment by exogenous CLE peptides plants in hydroponic conditions were used. All plants were grown at $21^{\circ} \mathrm{C}$ and $16 \mathrm{~h}$ photoperiod.

\section{Isolation of RsCLE genes}

Total DNA of $R$. sativus was isolated from radish seedlings using the cetyltrimethylammonium bromide (CTAB) method [42]. To amplify the fragments of radish $C L E$ genes, PCR was performed with primers designed for the conserve regions of corresponding genes of Arabidopsis thaliana and Brassica rapa using a VectorNTI Advance_10 (Invitrogen, United States) program (Additional file 4: Table S2). PCR-products were separated by electrophoresis in $1 \%$ agarose gel containing ethidium bromide $(0.1 \%)$. Target fragments were isolated from the gel using a Cleanup Mini Kit (Evrogen, Russia) according to the manufacturer's instructions and cloned into the pAL-TA vector (Evrogen, Russia). Transformation of chemically competent cells of Escherichia coli strain DH5 $\alpha$ was carried outaccording to the protocol described in [43]. Transformants were selected on solidified LB medium containing $100 \mathrm{mg} / \mathrm{L}$ ampicillin and X-Gal. Plasmid DNA of selected transformant clones was isolated by Plasmid Miniprep Kit (Evrogen, Russia) and sequenced in SPbU Research Park, Center of Molecular and Cell Technologies. Sequence aligning of Arabidopsis and radish genes was performed using the AlignX program of VectorNTI Advance_10 (Invitrogen) software.

\section{qRT-PCR analysis}

Total RNA was extracted by Purezol reagent (Bio-Rad, USA), purified with chloroform, and precipitated with isopropanol. The RNA pellet was washed three times with $80 \%$ ethanol, dried under air flow in a laminar box, dissolved in sterile deionized water. RNA was treated with DNase (Syntol, Russia), subsequently purified with chloroform, reprecipitated with $0.3 \mathrm{M}$ sodium acetate in the presence of ethanol and dissolved in sterile deionized water. RNA concentration was measured using the NanoDrop 2000c UV spectrophotometer (Thermo Scientific, USA) at $260 \mathrm{~nm}$. For reverse transcription, $0.5 \mu \mathrm{g}$ of RNA were used in all samples. RNA reverse transcription was performed using "Synthesis of cDNA first chain kit, version with oligodT" (Silex-M, Russia) according to the enclosed protocol.

The qRT-PCR experiments were done on a CFX-96 real-time PCR detection system with $\mathrm{C} 1000$ thermal cycler (Bio-Rad, USA), and Eva Green intercalating dye was used for detection (Syntol, Russia). Primers for qRT-PCR were designed to amplify $150-220$ bp 
fragments. All reactions were performed in triplicate and averaged. Cycle threshold values were obtained with the accompanying software, and data were analyzed by $2^{-\Delta \Delta \mathrm{Ct}}$ method [44]. Relative expression was normalized against constitutively expressed ubiquitin (RsUBQ) and glyceroaldehyde-3-phosphate dehydrogenase $(R s G A P D H)$ genes [45]. Experiments were repeated three times with independent biological samples.

\section{Construction of vectors}

PCR-fragments amplified on $R$. sativus DNA with primers for full-length CDS of RsCLER2, 19 and 41 were cloned to pENTR/D-TOPO vector (Invitrogen, USA) and after that transferred to pB7WG2D vector (Ghent, Belgium) for overexpression using the LR Clonase enzyme (Invitrogen, USA). This vector contains GFP gene under constitutive promoter for transgenic organs selection.

Constructs were introduced into Agrobacterium rhizogenes MSU440 strain via electroporation using Eppendorf Eporator ${ }^{\circ}$ (Eppendorf, Germany).

Agrobacterium rhizogenes-mediated plant transformation A. rhizogenes-mediated plant transformation was performed as described previously [22]. To obtain sterile $R$. sativus seedlings, seeds were sterilized for $7 \mathrm{~min}$ with a mixture of $30 \%$ hydrogen peroxide and $95 \%$ ethanol (1:1), then washed with sterile distilled water and placed for germination on the Murashige-Skoog medium [46]. GFPpositive transgenic roots were selected using epifluorescent stereomicroscope Leica M205FA (Germany), all GFPnegative (nontransgenic) roots were cut off, and after that plants were placed into pots with vermiculite and cultivated at $21^{\circ} \mathrm{C}$ with a 16 -h photoperiod. After about 7 days, plants were transferred to pots with soil. After 30 days (rosette stage) roots were harvested and analyzed.

\section{Plant treatment with synthetic CLE peptides}

For treatment with synthetic CLE peptides, $R$. sativus plants were grown in hydroponic system as described in [47]. CLE2p, CLE19p and CLE41p were used to treat plants, and CLE41def peptide with substitution of conserved G6 residue and non-hydroxylated prolin residues was used as a control (Additional file 5: Table S3). Synthetic peptides CLE2p, CLE19p, CLE41p and CLE41def of $95 \%$ purities were obtained from ATG Service Gene (Russia). All peptides used were diluted to $10 \mathrm{mM}$ stock solutions and stored at $-20{ }^{\circ} \mathrm{C}$. For plant treatment, stock solutions were added to hydroponic medium to working concentration $10 \mu \mathrm{M}$, and plant were cultivated in the medium with CLE peptide for 7 days.

\section{Seedlings treatment with auxin and cytokinin}

For treatment with auxin or cytokinin, sterile $R$. sativus 7-day old seedlings were planted on solid MS medium supplied with $10 \mathrm{mM}$ of synthetic auxin 1Naphtaleneacetic acid (NAA) or synthetic cytokinin 6Benzylaminopurine (BAP), and grown for 0.5, 2, 24 and $48 \mathrm{~h}$. Seedlings planted on MS medium without hormones were used as a control.

\section{Histological analysis}

Roots of soil-growing $R$. sativus and $R$. raphanistrum plants at $7^{\text {th }}, 15^{\text {th, }}$ and $30^{\text {th }}$ days were fixed with $3 \%$ paraformaldehyde, $0.25 \%$ glutaraldehyde, $0.1 \%$ Tween$20,0.1 \%$ Triton X-100 in 1/3 MTSB by vacuum infiltration for $10 \mathrm{~min}$ with subsequent incubation overnight at $4{ }^{\circ} \mathrm{C}$. Samples were ethanol-dehydrated and embedded in agarose $(3 \%)$, and $50 \mu \mathrm{m}$ sections were prepared with a Leica Vibratome VT-1200S (Leica, Germany). Samples were stained with $0.05 \%$ wt/vol toluidine blue for $5 \mathrm{~s}$ and analyzed under Leica DM4000 microscope (Leica, Germany).

\section{Statistical methods}

Welch's unpaired $t$-test as implemented in $\mathrm{R}$ statistical environment (v. 3.0.2) was used to compare numbers of xylem elements. Gene expression across samples was compared using Welch's $t$-test as implemented is $\mathrm{R}$ (3.0.2). Normality of distributions of data was tested using Shapiro-Wilk test in R (3.0.2).

\section{Additional files}

Additional file 1: Table S1. Expression of RSCLE genes in the organs of Raphanus raphanistrum and Raphanus sativus 15-day old plants. (PDF $105 \mathrm{~kb}$ )

Additional file 2: Figure S1. Expression of RSCLE genes in different organs of seedling in Rapahnus sativus. Expression levels are shown relative to the expression of RSCLE1 found in the apex of 7-day old seedlings. Error bars indicate standard deviation of three technical repeats. (PDF $188 \mathrm{~kb}$ )

Additional file 3: Figure S2. Expression of RSCLE genes in hypocotyls of Rapahnus sativus and Raphanus sativus (line 27) at different stage of development. Expression levels are shown relative to the expression found in the hypocotyl of 7-day old seedlings. Error bars indicate standard deviation of three technical repeats. (PDF $186 \mathrm{~kb}$ )

Additional file 4: Table S2. Primers for identification of RSCLE genes. (PDF 179 kb)

Additional file 5: Table S3. Synthetic CLE peptides used for treatment of radish plants. (PDF $287 \mathrm{~kb}$ )

\section{Competing interests}

The authors declare that they have no competing interests.

\section{Authors' contributions}

MG constructed vectors for RSCLE genes overexpression, carried out A. rhizogenes-mediated plant transformation and histological researches, ID carried out cloning and qRT-PCR of RsCLE genes, ML treated radish plants with CLE peptides, VT and ID analyzed the data and drafted the manuscript, AT carried out statistical analyses and revised the manuscript, LL planned the experiments, supervised the research and revised the manuscript. All authors read and approved the manuscript.

\section{Declarations}

Publication of this article has been funded by the Russian Scientific

Foundation (Project No. 14-14-00161). 
This article has been published as part of BMC Plant Biology Volume 16 Supplement 1, 2015: Selected articles from PlantGen 2015 conference: Plant biology. The full contents of the supplement are available online at http://www.biomedcentral.com/bmcplantbiol/supplements/16/S1.

\section{Acknowledgments}

The work was supported by grants of Russian Foundation for Basic Researches 14-04-00591, 15-29-02737, and 15-34-20071, and grant of Saint-Petersburg state university 1.38.676.2013.

\section{Published: 27 January 2016}

\section{References}

1. Cock JM, McCormick S. A large family of genes that share homology with CLAVATA3. Plant Physiol. 2001;126:939-42.

2. Miyawaki K, Tabata R, Sawa S. Evolutionarily conserved CLE peptide signaling in plant development, symbiosis, and parasitism. Curr Opin Plant Biol. 2013;16:598-606.

3. Oelkers K, Goffard N, Weiller GF, Gresshoff PM, Mathesius U, Frickey T. Bioinformatics analysis of the CLE signaling peptide family. BMC Plant Biol. 2008;8:1-15.

4. Wang XH, Mitchum MG, Gao BL, Li CY, Diab H, Baum TJ, et al. A parasitism gene from a plant-parasitic nematode with function similar to CLAVATA3/ESR (CLE) of Arabidopsis thaliana. Mol Plant Pathol. 2005;6:187-91.

5. Sharma VK, Ramirez J, Fletcher JC. The Arabidopsis CLV3-like (CLE) genes are expressed in diverse tissues and encode secreted proteins. Plant Mol Biol. 2003:51:415-25.

6. Jun J, Fiume E, Roeder AH, Meng L, Sharma VK, Osmont KS, et al. Comprehensive analysis of CLE polypeptide signaling gene expression and overexpression activity in Arabidopsis. Plant Physiol. 2010;54:1721-36.

7. Ito Y, Nakanomyo I, Motose H, Iwamoto K, Sawa S, Dohmae N, et al. Dodeca-CLE peptides as suppressors of plant stem cell differentiation. Science. 2006;313:842-45.

8. Whitford R, Fernandez A, De Groodt R, Ortega E, Hilson P. Plant CLE peptides from two distinct functional classes synergistically induce division of vascular cells. Proc Natl Acad Sci U S A. 2008;105:18625-30.

9. Fletcher JC, Brand U, Running MP, Simon R, Meyerowitz EM. Signaling of cell fate decisions by CLAVATA3 in Arabidopsis shoot meristems. Science. 1999;283:1911-14.

10. Hobe M, Müller R, Grünewald M, Brand U, Simon R. Loss of CLE40, a protein functionally equivalent to the stem cell restricting signal $C L V 3$, enhances root waving in Arabidopsis. Dev Genes Evol. 2003;213:371-81.

11. Hirakawa $Y$, Kondo $Y$, Fukuda $H$. Regulation of vascular development by CLE peptide-receptor systems. J Integr Plant Biol. 2010;52:8-16.

12. Mortier V, Den Herder G, Whitford R, Van de Velde W, Rombauts S, D'Haeseleer K, et al. CLE peptides control Medicago truncatula nodulation locally and systemically. Plant Physiol. 2010;153:222-37.

13. Osipova MA, Mortier V, Demchenko KN, Tsyganov VE, Tikhonovich IA, Lutova LA, et al. WUSCHEL-RELATED HOMEOBOX5 gene expression and interaction of CLE peptides with components of the systemic control add two pieces to the puzzle of autoregulation of nodulation. Plant Physiol. 2012;158:1329-41.

14. Fiume $\mathrm{E}$, Fletcher $\mathrm{C}$. Regulation of Arabidopsis embryo and endosperm development by the polypeptide signaling molecule CLE8. Plant Cell. 2012;24:1000-12.

15. Kondo Y, Hirakawa Y, Kieber JJ, Fukuda H. CLE peptides can negatively regulate protoxylem vessel formation via cytokinin signaling. Plant Cell Physiol. 2011;52:37-48.

16. Lewis-Jones $\sqcup$, Thorpe JP, Wallis GP. Genetic divergence in four species of the genus Raphanus: implications for the ancestry of the domestic radish R. sativus. Biol J Linn Soc. 1982;18:35-48.

17. Kaneko Y, Kimizuka-Takagi C, Bang SW, Matsuzawa Y. Radish. In: Kole C, editor. Genome Mapping and Molecular Breeding in Plants. New York: Springer; 2007. p. 141-60.

18. RadishBase. Michigan State University and J. Craig Venter Institute, USA. 2006. http://bioinfo.bti.cornell.edu/radish. Accessed 1 December 2013.

19. Shen D, Sun H, Huang M, Zheng Y, Li X, Fei Z. RadishBase: a database for genomics and genetics of radish. Plant Cell Physiol. 2013;54:e3. doi:10.1093/pcp/pcs176.

20. Shen D, Sun H, Huang M, Zheng Y, Qiu Y, Li X, et al. Comprehensive analysis of expressed sequence tags from cultivated and wild radish (Raphanus spp.). BMC Genomics. 2013;14:721. 21.
21. Buzovkina IS, Lutova LA. The genetic collection of radish inbred lines: history and prospects. Russ J Genet. 2007;43:1181-92.

22. Lebedeva MA, Tvorogova VE, Vinogradova AP, Gancheva MA, Azarakhsh M, Ilina EL, et al. Initiation of spontaneous tumors in radish (Raphanus sativus): Cellular, molecular and physiological events. J Plant Physiol. 2015;173:97-104.

23. Usuda H, Demura T, Shimogawara K, Fukuda H. Development of sink capacity of the "storage root" in a radish cultivar with a high ratio of "storage root" to shoot. Plant Cell Physiol. 2007:40:369-77.

24. Ting FST, Wren MJ. Storage organ development in radish (Raphanus sativus L.). 2. Effects of growth promoters on cambial activity in cultured roots, decapitated seedlings and intact plants. Ann Bot. 1980;46:277-84.

25. Zaki HEM, Takahata Y, Yokoi S. Analysis of the morphological and anatomical characteristics of roots in three radish (Raphanus sativus) cultivars that differ in root shape. J Hortic Sci Biotech. 2012;87:172.

26. Takano T. Studies on the pithiness of radish roots. IV. On the process of pithy tissue formation in the radish root. J Jap Soc hort Sci. 1966;35:152-7.

27. Esau K. Anatomy of seed plants. 2nd ed. New York: John Wiley \& Sons, Inc; 1977.

28. Brassica Genome Gateway. John Innes Centre, Norwich Research Park, Norwich, Norfolk UK. 2001. http://brassica.nbi.ac.uk/. Accessed 27 Sept 2013.

29. Song XF, Guo P, Ren SC, Xu TT, Liu CM. Antagonistic peptide technology for functional dissection of CLV3/ESR genes in Arabidopsis. Plant Physiol. 2013:161:1076-85.

30. Hirakawa $Y$, Shinohara $H$, Kondo $Y$, Inoue A, Nakanomyo I, Ogawa M, et al. Non-cell-autonomous control of vascular stem cell fate by a CLE peptide/ receptor system. Proc Natl Acad Sci U S A. 2008;105:15208-13.

31. Yaginuma $H$, Hirakawa $Y$, Kondo $Y$, Ohashi-Ito $K$, Fukuda $H$. A novel function of TDIF-related peptides: promotion of axillary bud formation. Plant Cell Physiol. 2011;52:1354-64.

32. Fiers M, Hause G, Boutilier K, Casamitjana-Martinez E, Weijers D, Offringa R, et al. Mis-expression of the CLV3/ESR-like gene CLE19 in Arabidopsis leads to a consumption of root meristem. Gene. 2004;327:37-49.

33. Strabala TJ, O'Donnell PJ, Smit AM, Ampomah-Dwamena C, Martin EJ, Netzler N, et al. Gain-of-function phenotypes of many CLAVATA3/ESR genes, including four new family members, correlate with tandem variations in the conserved CLAVATA3/ESR domain. Plant Physiol. 2006;140:1331-44.

34. Meng L, Feldman $\sqcup$. CLE genes may act in a variety of tissues/cells and involve other signaling cascades in addition to CLV3-WUS-like pathways. Plant Signal Behav. 2011;6:105-8.

35. Czyzewicz N, Shi CL, Vu LD, Van De Cotte B, Hodgman C, Butenko MA, et al. Modulation of Arabidopsis and monocot root architecture by CLAVATA3/EMBRYO SURROUNDING REGION 26 peptide. J Exp Bot. 2015:66:5229-43.

36. Bishopp A, Help H, El-Showk S, Weijers D, Scheres B, Friml J, et al. A mutually inhibitory interaction between auxin and cytokinin specifies vascular pattern in roots. Curr Biol. 2011;21:917-26.

37. Matsumoto-Kitano M, Kusumoto T, Tarkowski P, Kinoshita-Tsujimura K, Václavíková K, Miyawaki K, et al. Cytokinins are central regulators of cambial activity. Proc Natl Acad Sci U S A. 2008;105:20027-31.

38. Suer S, Agusti J, Sanchez P, Schwarz M, Greb T. WOX4 imparts auxin responsiveness to cambium cells in Arabidopsis. Plant Cell. 2011;23:3247-59.

39. Milhinhos A, Miguel CM. Hormone interactions in xylem development: a matter of signals. Plant Cell Rep. 2013;32:867-83.

40. Guo H, Zhang W, Tian H, Zheng K, Dai X, Liu S, et al. An auxin responsive CLE gene regulates shoot apical meristem development in Arabidopsis. Front Plant Sci. 2015;6:295. 1.

41. Donner TJ, Sherr I, Scarpella E. Auxin signal transduction in Arabidopsis vein formation. Plant Signal Behav. 2010;5:70-2.

42. Murray MG, Thompson WF. Rapid isolation of high molecular weight DNA Nucleic Acids Res. 1980;8:4321-5.

43. Inoue $\mathrm{H}$, Nojima $\mathrm{H}$, Okayama $\mathrm{H}$. High efficiency transformation of Escherichia coli with plasmids. Gene. 1990;96:23-8.

44. Livak KJ, Schmittgen TD. Analysis of relative gene expression data usingreal-time quantitative PCR and the 2(-Delta Delta C(T)) Method. Methods. 2001;25:402-8.

45. Dodueva IE, Kiryushkin SA, Osipova MA, Yurlova EV, Buzovkina IS, Lutova LA. Influence of cytokinins on the expression of CLE genes in radish. Russ J Plant Physiol. 2013;60:399-407.

46. Murashige T, Skoog F. A revised medium for rapid growth and bioassays with tobacco tissue cultures. Physiol Plant. 1962;15:473-97.

47. Maddison J, Lyons T, Plöchl M, Barnes J. Hydroponically cultivated radish fed L-galactono-1,4-lactone exhibit increased tolerance to ozone. Planta. 2002;214:383-91. 